\title{
ADICCIÓN A LA INTERNET Y AGRESIVIDAD EN ESTUDIANTES DE SECUNDARIA DEL PERÚ
}

\author{
INTERNET ADDICTION AND AGGRESSION IN HIGH SCHOOL STUDENTS \\ FROM PERU
}

\author{
M. Matalinares, C. Arenas, G. Díaz, A. Dioses, L. Yarlequé*, O. Raymundo, D. Baca, E. Fernández, J. Uceda, \\ Y. Huari, N. Villavicencio, P. Vargas, M. Quispe, E. Sánchez, V. Leyva, A. Diaz, A. Campos, y J. Yaringaño \\ UNMSM \\ (Recibido el 15/2/13 Aceptado el 14/5/13)
}

\section{RESUMEN}

El presente estudio tuvo como objetivo establecer la relación existente entre la agresividad y la adicción a la Internet, la muestra estuvo constituida por 2225 estudiantes de $3 .^{\text {ero }}$ a $5 .{ }^{\text {to }}$ año de secundaria de 13 ciudades representativas de la costa, sierra y selva del Perú, con edades entre 13 y 19 años. El diseño del estudio fue descriptivo correlacional y comparativo; y los instrumentos utilizados fueron el cuestionario de agresión, Buss y Perry (1992) y el test de adicción a la Internet, Young (1998), se tomaron ambas versiones adaptadas a la realidad nacional. Los resultados demuestran que existe una relación positiva entre agresividad y adicción a la Internet, así como entre todos los componentes de ambas variables, a nivel comparativo se apreció que los varones tienden a ser más agresivos física y verbalmente, y las mujeres presentan más ira y hostilidad, los factores de riesgo para desarrollar una adicción a la Internet serían el género masculino, vivir en la costa del Perú, por el fácil acceso $\mathrm{t}$ sin supervisión, tener entre 15 y 16 años y presentar una alta agresividad, estos factores pueden ayudar a adquirir, desarrollar y mantener una adicción al Internet.

Palabras clave: adicción, adolescentes, agresividad, Internet

\begin{abstract}
Present study aimed to identify the relationship between aggression and Internet addiction. Sample consisted of 2225 high school students, aged 13 to 19, from third to fifth years. They came from 13 cities representing coast, highlands and jungle Peruvian regions. A comparative and correlational descriptive study design was used and the Buss \& Perry Hostility Inventory (1992) and the Internet Addiction Test by K. Young were the instruments applied, previously adapted to national reality. Results show positive relationship between Internet addiction and aggression and among all components of both research variables. Men tend to be more physically and verbally aggressive while women tend to experience more anger and hostility. Male gender, living on the coast region of Peru, easier access to Internet without supervision, being between 15 and 16 years old and having high aggression scores were risk- factors found for developing addiction to Internet. Implications of the study are discussed.
\end{abstract}

Keywords: addiction, adolescents, aggression, Internet 


\section{INTRODUCCIÓN}

La Internet ha comenzado a formar parte de la vida diaria de la mayoría de los individuos en el mundo, pero con su desarrollo y gran crecimiento han aparecido también los primeros casos de psicopatología relacionados con la red. El trastorno impulsivo compulsivo por el uso de Internet ha sido conocido con muchos nombres a lo largo del tiempo: desorden de adicción a Internet (Goldberg, 1995, citado por Balaguer 2001), uso compulsivo de Internet (Morahan y Schumacker, 1997), o uso patológico de Internet (Young y Nabuco, 2011). Estas diversas denominaciones que se le han asignado, responden a una dificultad en la conceptualización diagnóstica que estos comportamientos desadaptativos han acarreado durante los últimos años, tomando en cuenta que hace 15 años comenzaban a aparecer casos de adicción a la red en Estados Unidos, los mismos que fueron estudiados por Young (1998), quien considera al Internet como un potencial ente adictivo en el ser humano.

En la actualidad, por las repercusiones que acarrea y su alta incidencia social, sobre todo en países como China, Korea y Taiwán, la también denominada adicción al Internet, se va encontrar en la revisión del DSM V conceptualizada como un trastorno impulsivo compulsivo por el uso de Internet, y todas sus variantes (juego excesivo, preocupaciones sexuales y mensajería) comparten estos cuatro componentes: el uso excesivo, la retirada, tolerancia y consecuencias negativas (Beard y Wolf, 2001, citado por Block, 2008).

A nivel internacional, han surgido diversos estudios que sustentan el potencial adictivo de las redes para los individuos, donde encontramos investigadores como Kraut y cols. (1998) citados por Sánchez e Iruarrizaga (2009) quienes examinaron el impacto del Internet sobre 169 personas en 73 hogares, encontrando que un gran uso de Internet estaba asociado con un decremento en la comunicación con los miembros de la familia en el hogar, así como en el tamaño de su círculo social y un incremento en su depresión y soledad. Yen, Ko, Yen, Chen, Chung y Chen (2008) trabajaron con 3662 alumnos de ambos sexos, a quienes aplicaron escalas para evaluar los síntomas psiquiátricos, la adicción a Internet y el uso de sustancias. Los resultados mostraron que la adicción a Internet y a las sustancias en adolescentes se asocia con graves síntomas psiquiátricos. La hostilidad y la depresión también estaban asociadas con la adicción a Internet y al uso de sustancias. Un estudio más reciente hecho por Timothy Liu, citado por la Agencia Reuters (2011), investigador de la Universidad de Yale, quien realizó una investigación con 3,500 alumnos de secundaria en la ciudad Connecticut, encontró que uno de cada 25 adolescentes manifestaron tener una "necesidad acuciante" de estar en Internet o aseguraron estar en tensión cuando no estaban online, o indicaron que habían intentado reducir o cortar del todo el tiempo que pasaban en la red sin éxito. Lo que demuestra también este estudio es que los estudiantes con un "uso problemático de Internet" eran más vulnerables que sus compañeros a estar deprimidos o agresivos, y a consumir drogas. Ante lo mencionado, se resalta a la agresividad como un aspecto importante en la adicción a Internet, considerando que Berkowitz (1996, citado por Matalinares, Arenas, Sotelo, Díaz, Dioses, 
Yaringaño, Muratta, Pareja, Tipacti, 2010) define a la agresividad como un estado emocional que consiste en sentimientos de odio y deseos de dañar a otra persona, animal u objeto. En cambio, Buss (1992) no solo ve a la agresividad como un estado emocional, sino considera que las respuestas agresivas poseen dos características: la descarga de estímulos nocivos y un contexto interpersonal; definiendo a la agresión como una reacción que descarga estímulos nocivos sobre otros organismos, lo cual exacerba las consecuencias sociales, ya que el Internet podría ser considerado como un contexto interpersonal novedoso y útil frente a la agresividad de los adolescentes.

La agresividad, por ser nociva y dañina fue estudiada en diferentes contextos y por diversos autores, dentro de los cuales se puede observar a investigadores como Castrillon y Vieco (2002), quienes realizaron un estudio en la ciudad de Medellín, donde encontraron que hay diferencias de género en la expresión de la agresión, ya que los hombres tienden a manifestar más una agresión física y directa, mientras que las mujeres optan por una agresión verbal e indirecta. Asimismo, Muñoz (2002) realizó un estudio sobre la adolescencia y agresividad, reportando que los adolescentes agresivos presentan déficits cognitivos en el procesamiento de la información social, tanto en situaciones hipotéticas, como en situaciones reales vividas por los propios adolescentes, en el razonamiento moral presentan un estadio menos desarrollado, así como manifiestan una menor valoración de la solidaridad y baja autoestima. Respecto a la percepción autobiográfica, los agresivos perciben mayores dificultades de relación y de comunicación en los tres contextos donde se desarrollan: familia, escuela y grupo de iguales.

En el Perú, aún hay pocas investigaciones relacionadas a la adicción a Internet, sin embargo durante los últimos años el interés científico en este tema está motivando su estudio, ya que se puede encontrar a Cruzado, Matos, Kendall (2006), quienes realizaron un Perfil clínico y Epidemiológico de pacientes adictos a Internet, hospitalizados en un Instituto Nacional de Salud Mental, así como estimaron que el Perú era para el año 2005 el segundo en Latinoamérica en cuanto a tasa de penetración de Internet (11,5 usuarios/100 habitantes) y el $66 \%$ de adolescentes de Lima accedían a Internet regularmente, la mayoría a través de cabinas públicas. Asimismo se han realizado estudios como los de Quispe (2011) quien trabajó en la adaptación y validación del test de adicción al Internet en una muestra de alumnos de 13 hasta 18 años de Lima. Así como Lam, Contreras, Mori, Nizama y otros (2011) validaron un instrumento para evaluar la adicción al Internet en escolares adolescentes de Lima. En cuanto a la agresividad podemos encontrar trabajos como el de Matalinares et al. (2010) donde investigaron el clima familiar y la agresividad en estudiantes de secundaria, encontrando que la agresividad de los estudiantes se muestra diferente en función del sexo. No obstante, el estudio sobre la relación entre la adicción a Internet y la agresividad en el Perú apenas se ha iniciado. 
Adicción a Internet y AgResividad en estudiantes de secundaria del Perú

\section{HIPÓTESIS}

\section{Hipótesis general}

La adicción a la Internet se relaciona positivamente con la agresividad en estudiantes de secundaria de diversas regiones del Perú.

\section{Hipótesis específica}

Los componentes de la adicción al Internet se relacionan positivamente con los factores de agresividad en los estudiantes de secundaria de diversas regiones del Perú.

Existen diferencias significativas entre la adicción a la Internet y la agresividad en los estudiantes de las diversas regiones del Perú, según sexo, procedencia y edad.

\section{MÉTODO}

\section{Tipo y diseño de investigación}

En concordancia con las hipótesis formuladas se empleó en un primer momento el método descriptivo con un diseño correlacional para relacionar las dos variables, y luego el diseño descriptivo comparativo para comparar los resultados de los estudiantes de las diversas regiones del Perú, en función al sexo, procedencia y edad.

\section{Sujetos}

La muestra estuvo constituida por 2225 estudiantes de 3 ero $^{\text {e }} 5 .^{\text {to }}$ año de secundaria de 13 ciudades representativas de la costa, sierra y selva del Perú (Piura, Chiclayo, Trujillo, Lima, Tacna, Huánuco, Huancayo, Ayacucho, Huancavelica, Cuzco, San Martín, Amazonas y Pucallpa), de los cuales un 36\% tenían entre 13 y 14 años, el $55.96 \%$ entre 15 y 16 años, mientras que un $7.69 \%$ de 17 a 19 años, en cuanto a la distribución por género se aprecia que el $52.9 \%$ son mujeres y el $47.10 \%$ varones.

Cabe resaltar que los estudiantes tienen un mayor acceso a Internet desde una cabina con un $59.1 \%$ en varones y $55.7 \%$ en mujeres, y el tiempo de permanencia en Internet es de 6 a 10 horas o más de 10 horas a la semana en un porcentaje de $32.6 \%$ de varones y $25.1 \%$ de mujeres. En cuanto a los motivos por los que usan el Internet, el porcentaje de adolescentes que atribuyen que el Internet es importante para conocer personas son un $66.7 \%$ de varones y un $51 \%$ de mujeres, para tener espacio personal el $72 \%$ de varones y $70.3 \%$ de mujeres, para buscar información para adultos el $31.8 \%$ de varones y el $23.3 \%$ de mujeres, y para jugar en red el $73.1 \%$ de varones y $42.8 \%$ de mujeres.

\section{Técnicas e instrumento}

Para el presente estudio se utilizó la técnica psicométrica y de observación indirecta.

Los instrumentos utilizados fueron: 
1. Test de adicción al Internet (TAI), autora: K. Young (1998), estandarizado por Raymundo, Matalinares y Baca (2012, en prensa). Su administración puede ser individual o colectiva, el tiempo de aplicación es en promedio 20 minutos. El test evalúa los indicadores de adición al Internet distribuidos en cuatro componentes: el uso excesivo, la retirada, tolerancia y consecuencia negativas, la tipificación se realizó mediante baremos nacionales según el sexo y lugar de procedencia (costa, sierra y selva).

La confiabilidad total del test es de 0.870 , así como la confiabilidad por Costa es de 0.876 , sierra 0.878 y selva con 0.836 . En el análisis factorial exploratorio obtuvieron cuatro componentes: tolerancia, uso excesivo, retirada y consecuencias negativas, con una varianza de $49.57 \%$. Estos componentes fueron ratificados mediante el análisis factorial confirmatorio.

El test de adicción al Internet adaptado al Perú, contempla los criterios diagnósticos del DSM -V para el trastorno impulsivo compulsivo por el uso de Internet, presenta los siguientes cuatro componentes:

a. El uso excesivo: Está asociado con una pérdida del sentido del tiempo, como una característica impulsiva del inicio del problema, donde la persona no logra controlar la necesidad de estar conectado al Internet, dejando de lado actividades sociales y familiar por pensar y estar conectado en línea, pasando más tiempo del que se había programado.

b. La tolerancia: Es la necesidad de un mejor equipamiento informático, un nuevo programa, o más horas de uso, como una reacción compulsiva para aliviar la ansiedad de no estar conectado, o ansiedad por situaciones estresantes de la vida cotidiana, las mismas que son reemplazadas por el uso del Internet.

c. La retirada o abstinencia: Se aprecian sentimientos de ira, ansiedad, y/o depresión cuando la computadora es inaccesible, interfiriendo en sus pensamientos, emociones y motivaciones, reduciendo el intervalo de tiempo de un acceso a otro a Internet.

d. Consecuencias negativas: Se aprecian argumentos, mentiras, bajo rendimiento, aislamiento social, fatiga y descuido en las actividades domésticas como consecuencia de estar conectado al Internet.

2. Cuestionario de agresión, creado por Buss y Perry (1992), adaptado por Matalinares, Yaringaño, Uceda, Fernández, Huari, Campos y Villavicencio (2012, en prensa) en una muestra de 3632 adolescentes de 10 a 19 años de 1 . ro al $5 .{ }^{\text {to }}$ de secundaria, procedentes de instituciones educativas de la costa, sierra y selva del Perú, el cuestionario de 29 preguntas obtuvo un alfa de Crombach del 0.836, ello demuestra que el test tiene un ajuste aceptable al modelo de cuatro factores, cabe resaltar que el tiempo de aplicación es de 20 minutos y puede ser tomado de forma individual o colectiva, las dimensiones presentes son: agresión física, agresión verbal, hostilidad e ira. 
El cuestionario de agresión adaptado al Perú, presenta los siguientes cuatro componentes:

- Agresión Física. Suele ser concebida como una respuesta adaptativa que forma parte de las estrategias de afrontamiento de los seres humanos a las amenazas externas. La agresión conlleva la aparición de comportamientos destructivos, lesivos o punitivos dirigidos a otras personas u objetos.

- Agresividad verbal. Se manifiesta en la forma imperativa e inapropiada con que el sujeto defiende sus derechos y trata de imponer, a la fuerza, sus puntos de vista, sentimientos e ideas, de manera directa o indirecta. Formas comunes de agresión verbal: insinuación maliciosa, ironía, burla, sarcasmo, agravio, mofa, ridiculización, afrenta, menosprecio, descalificación, humillación, escarnio, insulto, ofensa, injuria, calumnia, difamación, ultraje, etc.

- Hostilidad. Se refiere a la evaluación negativa acerca de las personas y las cosas (Buss, 1961). Se expresa hostilidad cuando decimos que alguien nos disgusta, especialmente si deseamos el mal para esta persona.

- Ira. El conjunto de sentimientos que siguen a la percepción de haber sido dañado, hace referencia principalmente a un conjunto de sentimientos que surgen de reacciones psicológicas internas y de las expresiones emocionales involuntarias producidas por la aparición de un acontecimiento desagradable (Berkowitz, 1996).

\section{Procedimientos}

La recolección de la información se efectuó de manera colectiva, en 13 ciudades representativas del Perú (Piura, Chiclayo, Trujillo, Lima, Tacna, Huánuco, Huancayo, Ayacucho, Huancavelica, Cuzco, San Martín, Amazonas y Pucallpa), siguiendo las normas establecidas en los respectivos manuales.

Las respuestas fueron calificadas en forma manual y se hizo una base de datos que fue procesada mediante el paquete estadístico SPPS versión 18.

\section{RESULTADOS}

\section{Análisis descriptivo}

Tabla 1. Descripción del acceso y uso del Internet

\begin{tabular}{cccccc}
\hline & \multicolumn{2}{c}{ Masculino } & \multicolumn{2}{c}{ Femenino } \\
\hline & $\mathrm{N}^{\circ}$ & $\%$ & $\mathrm{~N} .^{\circ}$ & $\%$ \\
\hline ¿Dónde tienes acceso a Internet? & & & & \\
\hline Casa & 224 & 21.4 & 353 & 30.0 \\
Cabina & 619 & 59.1 & 656 & 55.7
\end{tabular}


Matalinares, M., Arenas, C., Diaz, G., Dioses, A., Yarlequé, L., Raymundo, O., Baca, D., Fernández, E., et al.

\begin{tabular}{|c|c|c|c|c|c|}
\hline & Casa y Cabina & 205 & 19.6 & 168 & 14.3 \\
\hline \multicolumn{6}{|c|}{ ¿Cuánto tiempo usas Internet? } \\
\hline & $\begin{array}{l}\text { Menos de } 5 \text { horas sema- } \\
\text { nales }\end{array}$ & 706 & 67.4 & 881 & 74.9 \\
\hline & $\begin{array}{l}\text { Entre } 6 \text { a } 10 \text { horas se- } \\
\text { manales }\end{array}$ & 213 & 20.3 & 206 & 17.5 \\
\hline & $\begin{array}{l}\text { Más de } 10 \text { horas sema- } \\
\text { nales }\end{array}$ & 129 & 12.3 & 90 & 7.6 \\
\hline \multicolumn{6}{|c|}{ ¿Cuáles son los motivos importantes por los cuales usas Internet? } \\
\hline \multirow{3}{*}{$\begin{array}{l}\text { Para conocer } \\
\text { personas }\end{array}$} & Nada importante & 348 & 33.2 & 577 & 49.0 \\
\hline & Más o menos importante & 558 & 53.2 & 514 & 43.7 \\
\hline & Muy importante & 142 & 13.5 & 86 & 7.3 \\
\hline \multirow{3}{*}{$\begin{array}{l}\text { Para tener espacio } \\
\text { personal }\end{array}$} & Nada importante & 294 & 28.1 & 350 & 29.7 \\
\hline & Más o menos importante & 525 & 50.1 & 555 & 47.2 \\
\hline & Muy importante & 229 & 21.9 & 272 & 23.1 \\
\hline \multirow{3}{*}{$\begin{array}{l}\text { Para buscar } \\
\text { información para } \\
\text { adultos }\end{array}$} & Nada importante & 716 & 68.3 & 902 & 76.6 \\
\hline & Más o menos importante & 243 & 23.2 & 223 & 18.9 \\
\hline & Muy importante & 89 & 8.5 & 52 & 4.4 \\
\hline \multirow{3}{*}{ Para jugar en red } & Nada importante & 280 & 26.7 & 673 & 57.2 \\
\hline & Más o menos importante & 536 & 51.1 & 420 & 35.7 \\
\hline & Muy importante & 232 & 22.1 & 84 & 7.1 \\
\hline Total & & 1048 & 100.0 & 1177 & 100.0 \\
\hline
\end{tabular}

Se observa en la Tabla 1, que los alumnos acceden al Internet principalmente a través de una cabina (59.1\% de varones y $55.7 \%$ de mujeres), siendo el tiempo de mayor uso menor de 05 horas semanales $(67.4 \%$ de varones y $74.9 \%$ de mujeres). Entre los motivos más importantes por los cuales se accede se encontró: para conocer personas $(66.7 \%$ de varones y $51 \%$ de mujeres), para tener espacio personal (72\% de varones y $70.3 \%$ de mujeres), para buscar información para adultos (31.8\% en varones y $23.3 \%$ en mujeres), y para jugar en red $(73.1 \%$ de varones y $42.8 \%$ de mujeres). 
Adicción a Internet y AgResividad en estudiantes de secundaria del Perú

Tabla 2. Normalidad del Test de Adicción a Internet y Cuestionario de Agresión a través de Kolmogorov-Smirnov

\begin{tabular}{|c|c|c|c|c|c|c|c|}
\hline \multirow{2}{*}{$\begin{array}{l}\text { Instrumentos / } \\
\text { Componentes }\end{array}$} & \multicolumn{2}{|c|}{ Parámetros } & \multicolumn{3}{|c|}{ Diferencias extremas } & \multirow{2}{*}{$\begin{array}{c}\text { Kolmogorov- } \\
\text { Smirnov Z }\end{array}$} & \multirow{2}{*}{$\begin{array}{l}\text { Sig. (bi- } \\
\text { lateral) }\end{array}$} \\
\hline & Media & $\begin{array}{l}\text { Desviación } \\
\text { estándar }\end{array}$ & Absoluto & Positivo & Negativo & & \\
\hline $\begin{array}{l}\text { Cuestionario de } \\
\text { Agresión (AQ) }\end{array}$ & 74.60 & 15.984 & 0.021 & 0.020 & -0.021 & 0.967 & 0.307 \\
\hline $\begin{array}{l}\text { Agresividad } \\
\text { Física }\end{array}$ & 20.49 & 6.290 & 0.074 & 0.074 & -0.035 & 3.482 & 0.000 \\
\hline Hostilidad & 22.77 & 5.680 & 0.050 & 0.038 & -0.050 & 2.360 & 0.000 \\
\hline Ira & 19.37 & 4.663 & 0.052 & 0.048 & -0.052 & 2.441 & 0.000 \\
\hline $\begin{array}{c}\text { Agresividad } \\
\text { verbal }\end{array}$ & 11.98 & 3.645 & 0.064 & 0.064 & -0.053 & 3.000 & 0.000 \\
\hline $\begin{array}{l}\text { Test de adicción } \\
\text { al Internet (IAT) }\end{array}$ & 22.51 & 16.437 & 0.099 & 0.099 & -0.085 & 4.665 & 0.000 \\
\hline Tolerancia & 12.56 & 10.231 & 0.115 & 0.115 & -0.110 & 5.432 & 0.000 \\
\hline Uso excesivo & 5.50 & 4.024 & 0.120 & 0.120 & -0.086 & 5.672 & 0.000 \\
\hline Retirada & 2.19 & 2.006 & 0.194 & 0.194 & -0.137 & 9.173 & 0.000 \\
\hline $\begin{array}{c}\text { Consecuencias } \\
\text { negativas }\end{array}$ & 2.26 & 2.106 & 0.196 & 0.196 & -0.141 & 9.247 & 0.000 \\
\hline
\end{tabular}

La prueba de normalidad de Kolmogorov-Smirnov (ver Tabla 2) determinó utilizar pruebas no paramétricas para los cálculos inferenciales de ambas variables.

\section{Análisis inferencial}

Tabla 3: Correlaciones entre la variable Agresividad y la variable Adicción al Internet y sus componentes

\begin{tabular}{ccccccc}
\hline & & $\begin{array}{c}\text { Adicción } \\
\text { al Internet }\end{array}$ & Tolerancia & $\begin{array}{c}\text { Uso } \\
\text { excesivo }\end{array}$ & Retirada & $\begin{array}{c}\text { Consecuencias } \\
\text { negativas }\end{array}$ \\
\hline \multirow{2}{*}{ Agresividad } & $\begin{array}{c}\text { Rho } \\
\text { Sig. (bi- } \\
\text { lateral) } \\
\text { N. }{ }^{\circ}\end{array}$ & $.408(* *)$ & $.403(* *)$ & $.351(* *)$ & $.295(* *)$ & $.283(* *)$ \\
& 2225 & 2225 & 2225 & 2225 & .000 \\
\hline
\end{tabular}


Matalinares, M., Arenas, C., Díaz, G., Dioses, A., Yarlequé, L., Raymundo, O., Baca, D., Fernández, E., et al.

\begin{tabular}{|c|c|c|c|c|c|c|}
\hline \multirow{3}{*}{$\begin{array}{l}\text { Agresividad } \\
\text { física }\end{array}$} & Rho & $.394(* *)$ & $.375(* *)$ & $.339(* *)$ & $.315(* *)$ & $.309(* *)$ \\
\hline & $\begin{array}{l}\text { Sig. (bi- } \\
\text { lateral) }\end{array}$ & .000 & .000 & .000 & .000 & .000 \\
\hline & $\mathrm{N}$ & 2225 & 2225 & 2225 & 2225 & 2225 \\
\hline \multirow{3}{*}{$\begin{array}{l}\text { Agresividad } \\
\text { verbal }\end{array}$} & Rho & $.339(* *)$ & $.337(* *)$ & $.281(* *)$ & $.244(* *)$ & $.238(* *)$ \\
\hline & $\begin{array}{l}\text { Sig. (bi- } \\
\text { lateral) }\end{array}$ & .000 & .000 & .000 & .000 & .000 \\
\hline & $\mathrm{N}$ & 2225 & 2225 & 2225 & 2225 & 2225 \\
\hline \multirow{3}{*}{ Hostilidad } & Rho & $.255(* *)$ & $.256(* *)$ & $.229(* *)$ & $.163(* *)$ & $.159(* *)$ \\
\hline & $\begin{array}{l}\text { Sig. (bi- } \\
\text { lateral) }\end{array}$ & .000 & .000 & .000 & .000 & .000 \\
\hline & $\mathrm{N}$ & 2225 & 2225 & 2225 & 2225 & 2225 \\
\hline \multirow{3}{*}{ Ira } & Rho & $.286(* *)$ & $.289(* *)$ & $.239(* *)$ & $.196(* *)$ & $.191(* *)$ \\
\hline & $\begin{array}{l}\text { Sig. (bi- } \\
\text { lateral) }\end{array}$ & .000 & .000 & .000 & .000 & .000 \\
\hline & $\mathrm{N}$ & 2225 & 2225 & 2225 & 2225 & 2225 \\
\hline
\end{tabular}

** La correlación es altamente significativa al nivel 0,01 (bilateral).

Se aprecia en la Tabla 3 que existen correlaciones altamente significativas $(\mathrm{p}<0.01)$ y directas entre las variables Agresividad y todas sus subescalas con la variable Adicción al Internet y todas sus dimensiones

Tabla 4: Prueba de U de Mann-Whitney para estimar si existen diferencias de las variables Agresividad y Adicción al Internet según sexo

\begin{tabular}{|c|c|c|c|c|c|c|}
\hline Variable & Grupo & $\mathrm{N} .^{\circ}$ & $\begin{array}{l}\text { Rango } \\
\text { promedio }\end{array}$ & $\begin{array}{c}\text { U de Mann- } \\
\text { Whitney }\end{array}$ & $\mathrm{Z}$ & $\begin{array}{l}\text { Sig. asin- } \\
\text { tót. }\end{array}$ \\
\hline \multirow{2}{*}{ Agresividad } & Masculino & 1048 & 1163.10 & \multirow{2}{*}{564239.50} & \multirow{2}{*}{-3.47} & \multirow{2}{*}{$0.00 * *$} \\
\hline & Femenino & 1177 & 1068.39 & & & \\
\hline \multirow{2}{*}{$\begin{array}{l}\text { Agresividad } \\
\text { física }\end{array}$} & Masculino & 1048 & 1282.12 & \multirow{2}{*}{439508.50} & \multirow{2}{*}{-11.73} & \multirow{2}{*}{$0.00 * *$} \\
\hline & Femenino & 1177 & 962.41 & & & \\
\hline \multirow{2}{*}{$\begin{array}{l}\text { Agresividad } \\
\text { verbal }\end{array}$} & Masculino & 1048 & 1167.48 & \multirow{2}{*}{559657.00} & \multirow{2}{*}{-3.79} & \multirow{2}{*}{$0.00 * *$} \\
\hline & Femenino & 1177 & 1064.49 & & & \\
\hline \multirow{2}{*}{ Hostilidad } & Masculino & 1048 & 1051.97 & \multirow{2}{*}{552785.00} & \multirow{2}{*}{-4.23} & \multirow{2}{*}{$0.00 * *$} \\
\hline & Femenino & 1177 & 1167.34 & & & \\
\hline
\end{tabular}


Adicción a Internet y agresividad en estudiantes de secundaria del Perú

\begin{tabular}{|c|c|c|c|c|c|c|}
\hline \multirow{2}{*}{ Ira } & Masculino & 1048 & 1081.77 & \multirow{2}{*}{584022.50} & \multirow{2}{*}{-2.17} & \multirow{2}{*}{$0.03 *$} \\
\hline & Femenino & 1177 & 1140.80 & & & \\
\hline \multirow{2}{*}{$\begin{array}{l}\text { Adicción al } \\
\text { Internet }\end{array}$} & Masculino & 1177 & 1239.81 & \multirow{2}{*}{483853.50} & \multirow{2}{*}{-8.79} & \multirow{2}{*}{$0.00 * *$} \\
\hline & Femenino & 1048 & 1000.09 & & & \\
\hline \multirow{2}{*}{ Tolerancia } & Masculino & 1048 & 1226.14 & \multirow{2}{*}{498174.00} & \multirow{2}{*}{-7.84} & \multirow{2}{*}{$0.00 * *$} \\
\hline & Femenino & 1177 & 1012.26 & & & \\
\hline \multirow{2}{*}{ Uso Excesivo } & Masculino & 1048 & 1228.22 & \multirow{2}{*}{495999.50} & \multirow{2}{*}{-8.01} & \multirow{2}{*}{$0.00 * *$} \\
\hline & Femenino & 1177 & 1010.41 & & & \\
\hline \multirow{2}{*}{ Retirada } & Masculino & 1048 & 1220.88 & \multirow{2}{*}{503688.50} & \multirow{2}{*}{-7.61} & \multirow{2}{*}{$0.00 * *$} \\
\hline & Femenino & 1177 & 1016.94 & & & \\
\hline \multirow{2}{*}{$\begin{array}{c}\text { Consecuencias } \\
\text { Negativas }\end{array}$} & Masculino & 1048 & 1224.67 & \multirow{2}{*}{499720.50} & \multirow{2}{*}{-7.87} & \multirow{2}{*}{$0.00 * *$} \\
\hline & Femenino & 1177 & 1013.57 & & & \\
\hline
\end{tabular}

$* \mathrm{p}<0.05, * * \mathrm{p}<0.01$

En la Tabla 4 se observa que existen diferencias significativas $(\mathrm{p}<0.01)$ de la variable Agresividad y sus sub-escalas Agresividad Física y Agresividad Verbal a favor del sexo masculino; asimismo, en las sub-escalas Hostilidad e Ira existen diferencias muy significativas $(\mathrm{p}<0.01)$ y significativas $(\mathrm{p}<0.05)$, respectivamente, siendo la media de las mujeres más alta que la de los varones. De otro lado, se aprecia diferencias altamente significativas $(\mathrm{p}<0.01)$ de la variable Adicción al Internet y sus dimensiones observándose que los hombres puntuaron más alto que las mujeres en uso excesivo, tolerancia, retirada y consecuencias negativas.

Tabla 5. Prueba de Kruskal-Wallis de muestras independientes para estimar si existen diferencias de las variables Agresividad y Adicción al Internet según procedencia

\begin{tabular}{lcccccr}
\hline \multicolumn{1}{c}{ Variable } & Grupo & N. & $\begin{array}{c}\text { Rango } \\
\text { promedio }\end{array}$ & $\begin{array}{c}\text { U de Mann- } \\
\text { Whitney }\end{array}$ & Z & Sig. asintót. \\
\hline \multirow{2}{*}{ Agresividad } & Costa & 888 & 1094.41 & & & \\
& Sierra & 812 & 1143.38 & 2.87 & 2.00 & 0.24 \\
& Selva & 525 & 1097.44 & & & \\
\hline \multirow{2}{*}{ Agresividad física } & Costa & 888 & 1107.09 & & & $0.01^{*}$ \\
& Sierra & 812 & 1157.76 & 8.50 & 2.00 & \\
\hline
\end{tabular}


Matalinares, M., Arenas, C., Diaz, G., Dioses, A., Yarlequé, L., Raymundo, O., Baca, D., Fernández, E., et al.

\begin{tabular}{|c|c|c|c|c|c|c|}
\hline \multirow{3}{*}{ Agresividad verbal } & Costa & 888 & 1105.49 & \multirow{3}{*}{0.69} & \multirow{3}{*}{2.00} & \multirow{3}{*}{0.71} \\
\hline & Sierra & 812 & 1127.81 & & & \\
\hline & Selva & 525 & 1102.78 & & & \\
\hline \multirow{3}{*}{ Hostilidad } & Costa & 888 & 1082.10 & \multirow{3}{*}{3.58} & \multirow{3}{*}{2.00} & \multirow{3}{*}{0.17} \\
\hline & Sierra & 812 & 1139.05 & & & \\
\hline & Selva & 525 & 1124.96 & & & \\
\hline \multirow{3}{*}{ Ira } & Costa & 888 & 1102.40 & \multirow{3}{*}{1.05} & \multirow{3}{*}{2.00} & \multirow{3}{*}{0.59} \\
\hline & Sierra & 812 & 1108.73 & & & \\
\hline & Selva & 525 & 1137.51 & & & \\
\hline \multirow{3}{*}{ Adicción a Internet } & Costa & 888 & 1199.49 & \multirow{3}{*}{26.84} & \multirow{3}{*}{2.00} & \multirow{3}{*}{$0.00 * *$} \\
\hline & Sierra & 812 & 1052.91 & & & \\
\hline & Selva & 525 & 1059.63 & & & \\
\hline \multirow{3}{*}{ Tolerancia } & Costa & 888 & 1170.37 & \multirow{3}{*}{11.90} & \multirow{3}{*}{2.00} & \multirow{3}{*}{$0.00 * *$} \\
\hline & Sierra & 812 & 1070.46 & & & \\
\hline & Selva & 525 & 1081.73 & & & \\
\hline \multirow{3}{*}{ Uso excesivo } & Costa & 888 & 1245.91 & \multirow{3}{*}{67.21} & \multirow{3}{*}{2.00} & \multirow{3}{*}{$0.00 * *$} \\
\hline & Sierra & 812 & 998.22 & & & \\
\hline & Selva & 525 & 1065.71 & & & \\
\hline \multirow{3}{*}{ Retirada } & Costa & 888 & 1189.44 & \multirow{3}{*}{24.36} & \multirow{3}{*}{2.00} & \multirow{3}{*}{$0.00 * *$} \\
\hline & Sierra & 812 & 1085.02 & & & \\
\hline & Selva & 525 & 1026.96 & & & \\
\hline \multirow{3}{*}{$\begin{array}{l}\text { Consecuencias ne- } \\
\text { gativas }\end{array}$} & Costa & 888 & 1173.72 & \multirow{3}{*}{14.87} & \multirow{3}{*}{2.00} & \multirow{3}{*}{$0.00 * *$} \\
\hline & Sierra & 812 & 1087.89 & & & \\
\hline & Selva & 525 & 1049.10 & & & \\
\hline
\end{tabular}

$* \mathrm{p}<0.05, * * \mathrm{p}<0.01$

De acuerdo a la Tabla 5, se observa que existen diferencias significativas $(\mathrm{p}<0.05)$ de la sub-escala Agresividad Física según procedencia a favor de la sierra donde es más notoria que en la selva y costa. Por otra parte, se observa diferencias altamente significativas $(\mathrm{p}<0.01)$ de la variable Adicción al Internet y sus dimensiones a favor de la costa. 
Adicción a Internet y AgResividad en estudiantes de secundaria del Perú

Tabla 6. Prueba de Kruskal-Wallis de muestras independientes para estimar si existen diferencias de las variables Agresividad y Adicción al Internet según edad

\begin{tabular}{|c|c|c|c|c|c|c|}
\hline Variable & Grupo & $\mathrm{N}$ & $\begin{array}{l}\text { Rango } \\
\text { promedio }\end{array}$ & $\begin{array}{l}\text { U de Mann- } \\
\text { Whitney }\end{array}$ & $\mathrm{Z}$ & $\begin{array}{l}\text { Sig. } \\
\text { asintót. }\end{array}$ \\
\hline \multirow{3}{*}{ Agresividad } & $13-14$ & 809 & 1069.05 & \multirow{3}{*}{6.82} & \multirow{3}{*}{2.00} & \multirow{3}{*}{$0.03 *$} \\
\hline & $15-16$ & 1245 & 1132.21 & & & \\
\hline & $17-19$ & 171 & 1180.97 & & & \\
\hline \multirow{3}{*}{ Agresividad Física } & $13-14$ & 809 & 1062.50 & \multirow{3}{*}{15.62} & \multirow{3}{*}{2.00} & \multirow{3}{*}{$0.00 * *$} \\
\hline & $15-16$ & 1245 & 1124.26 & & & \\
\hline & $17-19$ & 171 & 1269.92 & & & \\
\hline \multirow{3}{*}{ Agresividad Verbal } & $13-14$ & 809 & 1075.11 & \multirow{3}{*}{4.75} & \multirow{3}{*}{2.00} & \multirow{3}{*}{0.09} \\
\hline & $15-16$ & 1245 & 1138.08 & & & \\
\hline & $17-19$ & 171 & 1109.61 & & & \\
\hline \multirow{3}{*}{ Hostilidad } & $13-14$ & 809 & 1093.92 & \multirow{3}{*}{1.30} & \multirow{3}{*}{2.00} & \multirow{3}{*}{0.52} \\
\hline & $15-16$ & 1245 & 1126.53 & & & \\
\hline & $17-19$ & 171 & 1104.69 & & & \\
\hline \multirow{3}{*}{ Ira } & $13-14$ & 809 & 1090.03 & \multirow{3}{*}{1.68} & \multirow{3}{*}{2.00} & \multirow{3}{*}{0.43} \\
\hline & $15-16$ & 1245 & 1124.70 & & & \\
\hline & $17-19$ & 171 & 1136.39 & & & \\
\hline \multirow{3}{*}{ Adicción al Internet } & $13-14$ & 809 & 1063.93 & \multirow{3}{*}{7.43} & \multirow{3}{*}{2.00} & \multirow{3}{*}{$0.02 *$} \\
\hline & $15-16$ & 1245 & 1141.80 & & & \\
\hline & $17-19$ & 171 & 1135.44 & & & \\
\hline \multirow{3}{*}{ Tolerancia } & $13-14$ & 809 & 1078.95 & \multirow{3}{*}{3.58} & \multirow{3}{*}{2.00} & \multirow{3}{*}{0.17} \\
\hline & $15-16$ & 1245 & 1133.02 & & & \\
\hline & $17-19$ & 171 & 1128.28 & & & \\
\hline \multirow{3}{*}{ Uso excesivo } & $13-14$ & 809 & 1069.95 & \multirow{3}{*}{5.95} & & \\
\hline & $15-16$ & 1245 & 1140.42 & & 2.00 & 0.05 \\
\hline & 17-19 & 171 & 1116.97 & & & \\
\hline & $13-14$ & 809 & 1029.34 & & & \\
\hline Retirada & $15-16$ & 1245 & 1162.39 & 22.39 & 2.00 & $0.00 * *$ \\
\hline & 17-19 & 171 & 1149.15 & & & \\
\hline & $13-14$ & 809 & 1029.19 & & & \\
\hline $\begin{array}{c}\text { Consecuencias } \\
\text { ngativas }\end{array}$ & $15-16$ & 1245 & 1158.70 & 22.51 & 2.00 & $0.00 * *$ \\
\hline & $17-19$ & 171 & 1176.71 & & & \\
\hline
\end{tabular}

$* \mathrm{p}<0.05, * * \mathrm{p}<0.01$ 
Según la Tabla 6 existen diferencias significativas en Agresividad Física, respectivamente, a favor del rango de edad 17-19 años. De otra parte, se encontró diferencias significativas $(\mathrm{p}<0.05)$ de la variable Adicción al Internet a favor del rango de edad 14-15 años; $y$, diferencias altamente significativas $(p<0.01)$ de las dimensiones Retirada y Consecuencias Negativas a favor del rango de edad 15-16 años y 17-19 años, respectivamente.

\section{DISCUSIÓN DE RESULTADOS}

El presente estudio fue realizado a nivel nacional con estudiantes de 3ero a 5to año de secundaria de 13 ciudades representativas del Perú (Piura, Chiclayo, Trujillo, Lima, Tacna, Huánuco, Huancayo, Ayacucho, Huancavelica, Cuzco, San Martín, Amazonas y Pucallpa) con edades entre los 13 y 19 años, de los cuales el 52.9\% fueron mujeres y el $47.10 \%$ varones. Las características encontradas en los alumnos son las siguientes: Se puede apreciar que mas del $50 \%$ de adolescentes de ambos sexos, tienen un facil acceso al Internet desde una cabina, considerando que las horas de uso se pueden ir ampliando conforme no cuenten con límites y control de sus padres o del adulto responsable, estas dificultades pueden acarrerar que el adolescente se encuentre vulnerable a adquirir una adicción al Internet, estos datos son apoyados por Cruzado, Matos, y Kendall, (2006) quienes identificaron en un grupo de pacientes con adicción al Internet que el $80 \%$ tuvo historias de disfunción familiar, es decir que el facil acceso a Internet sin supervisión sumado a dificultades familiares son un factor predisponente y de riesgo para el adolescente. Por otro lado, el $32.6 \%$ de varones y $25.1 \%$ de mujeres acceden a Internet de 6 a 10 horas o mas de 10 horas a la semana, esto se respalda por el estudio realizado por Muñoz, Navarro y Ortega (2003) quienes refieren que los varones son los que dedican más tiempo a la semana en la utilización de cualquiera de los recursos de Internet, asimismo se puede apreciar que la mayor parte de los adolescentes un $67.4 \%$ en varones y el $74.9 \%$ en mujeres, usan el Internet menos de 05 horas semanales, lo cual indica que tienen un uso moderado de Internet, ello denota que no hay un uso patológico cercano a la adicción, sin embargo un $12.3 \%$ de hombres y el $7.6 \%$ de mujeres, ya presentan un uso significativo del Internet, por lo cual si estarían próximos a este tipo de psicopatología.

En cuanto a los motivos por los que usan el Internet, el $66.7 \%$ de varones y un 51\% de mujeres atribuyen que el Internet es importante para conocer personas, el $72 \%$ de varones y $70.3 \%$ de mujeres refiere utilizar el Internet para buscar un espacio personal, el $31.8 \%$ de varones y el $23.3 \%$ de mujeres usa el Internet para buscar información para adultos y el $73.1 \%$ de varones y $42.8 \%$ de mujeres le da importancia al uso de Internet para los juegos en red. Se puede deducir que un motivo significativo por el cual usan el Internet deviene a la búsqueda de privacidad, lo cual se asocia con la búsqueda de identidad personal de los adolescentes, y el interes social por interactuar con su grupo de pares, por ende se puede atribuir que la masificación del Internet en los ultimos años, ha generado un medio de comunicación, de interacción y de organización social diferente, sobre el que se basa una nueva forma de sociedad y en el que está creciendo toda una nueva generación, esta sociedad Castell (2001) la 
denominó "la sociedad red". Cabe resaltar que más del $70 \%$ de varones hace uso del Internet para jugar en red, por ello es importante detallar que estudios recientes demuestran que los sujetos con riesgo a desarrollar el uso patológico del Internet tienen niveles significativamente más altos de adicción a los juegos en línea, adicción al cibersexo, ideación suicida y el abuso de alcohol, esto reportado por Tsouvelas y Giotakos (2011), ante ello es importante mencionar que Muñoz, Navarro y Ortega (2003) identificaron que el $90 \%$ de los pacientes con adicción a Internet se abocaban prioritariamente a los juegos en red.

Al realizar el análisis correlacional se encontró una relación significativa directa entre la agresividad y la adicción al Internet, lo cual implica que ante un mayor comportamiento agresivo el adolescente tendrá una mayor posibilidad de ser adicto al Internet o viseversa. Ello se refuerza con estudios realizados por Carli et al. (2012), quienes informaron que de los 20 estudios analisados el 75\% reportaron correlaciones significativas del uso patológico del Internet con depresión, el 57\% con la ansiedad, el $100 \%$ con los síntomas del trastornos por déficit atencional e hiperactividad (TDAH), el 60\% con síntomas obsesivo compulsivos, y el 66\% con hostilidad / agresión. Así mismo en el estudio hecho por Cruzado, Matos y Kendall (2006) reportaron que los rasgos de personalidad más frecuentes en pacientes adictos a Internet fueron los antisociales (40\%), así como los síntomas principales fueron irritabilidad (80\%) y bajo del rendimiento académico (76.7\%). Así mismo uno de los estudios que nos permite entender este fenómeno en amplitud es el realizado por Yen, Wu, Huang y Ko (2011), quienes buscaron identificar las diferencias entre la hostilidad en el mundo real y virtual en jóvenes universitarios, sus resultados determinaron que los adictos a Internet presentaron una mayor hostilidad tanto en el mundo real como en el virtual, en comparación con quienes no presentaban adicción al Internet, asimismo aquellos jóvenes que usan el Internet principalmente para juegos en red tuvieron mayor hostilidad en su comportamiento, no solo en el mundo real sino también en el virtual. Ante los hallazgos reportados se puede apreciar que la adicción al Internet se asocia con problemas en la salud mental como son la depresión, agresividad y ansiedad, por ende el tener en cuenta la comorbilidad de este trastorno permitirá a los psicólogos intervenir y prevenir que los adolescentes adquieran conductas adictivas hacia el Internet.

En el análisis por componentes de la adicción al Internet, se obtuvo que ante una mayor tolerancia se aprecia una mayor agresividad física, verbal, hostilidad e ira en los adolescentes, ello implica que a más horas de uso, como una reacción compulsiva para aliviar la ansiedad de no estar conectado, o ansiedad por situaciones estresantes de la vida cotidiana, se estima que se vincula con la agresividad. En tal sentido, se contrasta con los hallazgos de Viñas, Juan, Villar, Caparros, Pérez y Cornella (2002), quienes identificaron una correlación positiva entre el uso de Internet y varios indicadores psicopatológicos como la depresión, ansiedad y alteraciones del sueño. Con relación al componente de uso excesivo, se obtuvo una correlación significativa y directa con la agresividad física y verbal, la hostilidad e ira, ello implica que ante una mayor pérdida del sentido del tiempo, como una característica impulsiva por el escaso control de la necesidad de estar conectado 
a Internet, se va evidenciar una alta agresividad, la misma que estaría relacionada a la pérdida de control conductual en el uso de la red. Esto se asocia al estudio realizado por Muñoz, Fernández, y Gómez (2010), quienes consideran que una conexión abusiva reduce la tensión emocional, mostrando algunos síntomas que pueden ser interpretados como tolerancia, abstinencia y pérdida de control. Ante ello sugieren que los problemas asociados con un uso excesivo de Internet son similares a los de otras adicciones conductuales y tecnológicas.

En cuanto al componente de retirada o abstinencia, se evidencia una relación directa significativa con la agresividad física y verbal, la hostilidad e ira. Ello denota que ante la presencia de sentimientos de ira, ansiedad, y/o depresión cuando la computadora es inaccesible, la agresividad física y verbal, hostilidad e ira se incrementan. Como se corrobora en el estudio de Muñoz, Gámez y Fernández (2008), quienes encontraron que en relación a los síntomas de abstinencia al tener que interrumpir o reducir el uso de Internet por cualquier circunstancia, el 9,8\% de los jóvenes reconocieron experimentar sentimientos de irritación; el 3,5\% manifestaron la presencia de pensamientos recurrentes anticipando la sensación de bienestar que experimentarían con la siguiente conexión; y el 1,8\% experimentaron ansiedad o agitación y la aparición de un deseo fuerte de conexión. El componente de consecuencias negativas obtuvo una relación directa y significativa con la agresividad física y verbal, hostilidad e ira. En tal sentido, ante un incremento en las mentiras, bajo rendimiento académico, aislamiento social, fatiga y descuido en las actividades domésticas como consecuencia de estar conectado al Internet, el adolescente presentará mayor agresividad. Lo cual se corrobora con lo hallado por Gracia, Vigo, Fernández y Marcó (2002), quienes identificaron que la pérdida de tiempo de trabajo o clase por estar conectado a Internet, se asociada a una mayor ansiedad y disfunción social, ya que acceden con frecuencia a servicios de chat y a páginas de contenido solo para adultos.

En el análisis diferencial de ambas variables, agresividad y adicción al Internet, según el sexo, se obtuvieron diferencias significativas en todas las subescalas. En la variable agresividad se aprecia que los varones tienden a ser más agresivos física y verbalmente que las mujeres, ello se respalda con lo referido por Matalinares, Arenas, Sotelo, Díaz, Dioses, Yaringaño, Muratta, Pareja y Tipacti (2010), quienes refieren que en diversos estudios los varones han demostrado tener mayor agresividad física que las mujeres, posiblemente relacionado a la perspectiva biológica, ya que el genotipo de una persona y otros correlatos biológicos del género pueden influir en la propensión a la conducta agresiva, mientras que las mujeres muestran un predominio en la hostilidad e ira. Por otro lado, en la variable adicción al Internet, los varones están más predispuesto a desarrollarla, ya que se considera que un nivel alto en agresividad es un factor que puede influir en la adquisición, desarrollo y mantenimiento de dicha adicción, por ende el género masculino se muestra más vulnerable que el femenino.

En las diferencias de ambas variables, según la procedencia, se aprecia que en la agresividad física los adolescentes procedentes de la sierra tienden a ser un poco más agresivos que los de la costa y selva, esto se podría relacionar con el factor 
cultural, ya que las costumbres y estilos de vida en las diversas regiones del Perú son diferentes y están influenciada por tradiciones y creencias. En cuanto a la variable adicción al Internet, se observa que los adolescentes procedentes de la costa del Perú tienden a estar más predispuestos a ser adictos al Internet, esto denota que los jóvenes de la costa en su mayoría cuentan con un fácil acceso al Internet, ya que muchos de ellos tienen Internet en casa y pueden hallar mayor cantidad de cabinas públicas en las ciudades de la costa, en comparación a sus coetáneos de la sierra y selva, por ende el fácil acceso al Internet sería otro factor de riesgo en su uso compulsivo. En relación a las diferencias encontradas según la edad, el grupo de riesgo son los adolescentes de 15 a 16 años, ya que muestran una mayor agresividad y uso del Internet.

En síntesis, el presente estudio resalta la relación significativa entre la agresividad y adiccion al Internet, considerando como un factor de riesgo al género masculino, con un fácil acceso al Internet, sin supervisión, y en edades entre 15 y 16 años. Asimismo diversos estudios han planteado la relación de la adicción al Internet no solo con la agresividad, sino también con la depresión, el trastorno por déficit atencional e hiperactividad y la ansiedad, estos rasgos pueden estar presentes en la adquisición, desarrollo y mantenimiento del trastorno impulsivo compulsivo del uso de Internet, el mismo que por el avance de la tecnología se puede constituir en un peligro real, por ello vale la pena considerar las actividades preventivas para tratar este fenómeno, incluyendo a los jóvenes y sus padres, ya que la Internet de por sí es un herramienta sumamente útil a la sociedad y las comunicaciones, pero quien lo usa compulsivamente es el ser humano.

\section{CONCLUSIONES}

Existe una relación positiva y significativa entre la adicción al Internet con la agresividad en estudiantes de secundaria de diversas regiones del Perú.

Existe relación positiva entre todos los componentes de la adicción al Internet con las subescalas de agresividad en los estudiantes de secundaria de diversas regiones del Perú.

Existen diferencias significativas en la adicción al Internet según sexo, ya que el género masculino esta más predispuesto a desarrollar una adicción al Internet a diferencia del sexo femenino.

Existen diferencias significativas en la adicción al Internet según el lugar de procedencia, ya que los adolescentes procedentes de la costa del Perú tienden a estar más predispuestos a ser adictos al Internet.

Existen diferencias significativas en la adicción al Internet según la edad, ya que el grupo etáreo de mayor predisposición a ser agresivos y adictos a Internet son adolescentes entre 15 y 16 años. 
Existen diferencias significativas en la agresividad según sexo, ya que los varones tienden a ser más agresivos física y verbalmente que las mujeres, mientras que ellas presentan más ira y hostilidad.

Existen diferencias significativas en la agresividad según el lugar de procedencia, ya que los adolescentes procedentes de la sierra tienden a manifestar mayor agresividad física a diferencia de los adolescentes de la costa y selva.

Existen diferencias significativas en la agresividad según la edad, ya que el grupo etáreo de mayor predisposición a ser agresivos son adolescentes entre 17 y 19 años.

\section{REFERENCIAS BIBLIOGRÁFICAS}

Agencia Reuters (2011). ¿Cuántos adolescentes tienen adicción al Internet? Entrevista realizada el día viernes 20 de mayo de 2011 11:34. Recuperado el 29 de noviembre En: http://es.reuters.com/article/topNews/idESMAE74J0CJ20110520

Tsouvelas, G., Giotakos, O. (2011). Internet use and pathological Internet engagement in a sample of college students, Psychiatrike, 22(3), pp. 221-230.

Balaguer, R. 2001, "La adicción a Internet". Fuente original: Adicción, Montevideo: Cuadernos de la Coordinadora de Psicólogos del Uruguay. Disponible en el Archivo del Observatorio para la CiberSociedad en http://www.cibersociedad.net/archivo/ articulo.php?art $=2$

Block, J.J. (2008). Issues for DSM V: Internet adicction. Am J Psychiatry, 165:306-7.

Buss, A. y Perry, M. (1992). Cuestionario de agresión. Journal of personality and social psychology, 63, pp. 452-459.

Carli, V. Durkee, T. Wasserman, D. Hadlaczky, G. Despalins, R. Kramarz, E. Wasserman, C. Sarchiapone, M. Hoven, C. Brunner, R. Kaess, M. (2012). The association between pathological Internet use and comorbid psychopathology: a systematic review. Psychopathology, Epub ahead of print.

Castells, M. (2001). Internet y la sociedad red. La lección inaugural del programa de doctorado sobre la sociedad de la información y el conocimiento. Universitá Oberta de Catalunya (UOC). Barcelona, España. Recuperado de: http://tecnologiaedu.us.es/ nweb/htm/pdf/106.pdf.

Castrillón, M. y Vieco, D. (2002). Actitudes justificativas del comportamiento agresivo y violento en estudiantes universitarios de la ciudad de Medellín, Colombia. Revista de la Facultad de Salud Pública, 20(2), pp. 51-66.

Cruzado, L., Matos L. y Kendall, R. (2006). Actividad física en adolescentes y su relación con Agresividad, impulsividad, Internet y videojuegos. Revista Med Hered, 17, (4) 196-203.

Kraut, R., Patterson, M., Lundmark, V., Kiesler, S., Mukopahyay, T., Scherlis, W. (1998). Internet paradox: A social technology that reduces social involvement and psychological well-being? American Psychologist, 53(9), 1017-1031.

Lam, N. Contreras, H. Mori, E. Nizama, M. Gutiérrez, C. Hinostroza, W. Torrejón, E. Hinostroza, R. Coaquira, E. y Hinostroza, W. (2011) Adicción a Internet: desarrollo 
y validación de un Instrumento en escolares adolescentes de Lima, Revista Peruana de Medicina Experimental y Salud Pública. 28(3):462-469.

Matalinares, M., Arenas, C., Sotelo, L., Diaz, G., Dioses, A., Yaringaño, J., Muratta, R., Pareja, C., Tipacti, R. (2010). Clima familiar y agresividad en estudiantes de secundaria de Lima Metropolitana, Revista IIPSI, 13(1), pp. 109-128.

Matalinares, M. Yaringaño, J., Uceda, J., Fernández, E., Huari, Y., Campos, A. y Villavicencio, N. (2012) Revista IIPSI (en prensa).

Morahan, J. y Schumacher P. (2000). Incidence and correlates of pathological Internet use among college students. Computers in Human Behavior, 16, 13-29.

Muñoz, M., Navarro, E., Ortega, N. (2003). Patrones de uso de Internet en población universitaria española. Adicciones, 15(2), pp. 137-144.

Muñoz, F. (2002) Adolescencia y agresividad. (Tesis Doctoral). Recuperado de http:// eprints.ucm.es/tesis/19972000/S/4/S4017401.pdf.

Muñoz, M. Gámez, L. Fernández, L. (2008). Indicadores de pérdida de control, tolerancia y abstinencia en relación al uso de Internet en universitarios. Psicología Clínica, Legal y Forense. 8, pp. 43-56.

Muñoz, M. Fernández, L. Gámez, M. (2010). Analysis of the indicators ol pathological Internet use in Spanish university students. The Spanish Journal of Psychology, 13(2), pp. 697-707.

Quispe, J. (2011). Adaptación del test de adicción en Lima Norte. (Tesis para optar al título de licenciatura), Universidad César Vallejo - Lima.

Raymundo, O. , Matalinares, M. y Baca, D. (2012) Revista IIPSI (en prensa).

Sánchez, S. y Iruarrizaga, I. (2009) Nuevas Dimensiones, Nuevas adicciones: La adicción al Sexo en Internet. Intervención Psicosocial Vol. 18 N. ${ }^{\circ} 3$ Recuperado el 29 de noviembre de 2011 en: http://scielo.isciii.es/scielo.php?script=sci_arttext\&pi $\mathrm{d}=$ S1132-05592009000300006

Viñas, F., Juan, J., Villar, E., Caparros, B., Pérez, I. y Cornella, M. (2002). Internet y psicopatología: las nuevas formas de comunicación y su relación con diferentes índices de psicopatología. Clínica y Salud, 13, pp. 235-256.

Yen, J., Ko, C., Yen, C., Chen, S., Chung, W., y Chen, C. (2008). Psychiatric symptoms in adolescents with Internet addiction: Comparison with substance use. Psychiatry and Clinical Neurosciences, 62, 9-16.

Yen, J., Yen, C., Wu, Y., Huang, C., Ko, C. (2011). Hostility in the real world and online: the effect of Internet Addiction, depression, and online activity, Cyberpsychol Behav Soc Netw, 14(11), pp. 649-655.

Young, K. (1998). Caught in the Net. New York: John Wiley y Sons. Inc.

Young, K. y Rodgers, R. (1998). Internet addiction: Personality traits associated with its development. Artículo presentado en el $69^{\text {th }}$ annual meeting of the Eastern Psychological Association.

Young, K. y Nabuco, C. (2011). Internet Addiction; a handbook and guide to evaluation and treatment. Canadá: Jhon Wiley y Sons, Inc. 
Matalinares, M., Arenas, C., Diaz, G., Dioses, A., Yarlequé, L., Raymundo, O., Baca, D., Fernández, E., et al.

ANEXO 1

Tabla 7. Descripción de la muestra

\begin{tabular}{lccc}
\hline & & $\mathrm{N} .{ }^{\circ}$ & $\%$ \\
\hline Edad & & & \\
\hline & $13-14$ & 809 & 36.36 \\
& $15-16$ & 1245 & 55.96 \\
& $17-19$ & 171 & 7.69 \\
\hline Sexo & & & \\
\hline & Masculino & 1048 & 47.10 \\
& Femenino & 1177 & 52.90 \\
\hline Total & & 2225 & 100.0 \\
\hline
\end{tabular}

